\title{
Weighted Regularization of Maxwell Equations - Computations in Curvilinear Polygons
}

\author{
Martin Costabel, Monique Dauge, Daniel Martin and Gregory Vial \\ IRMAR, Université de Rennes 1, Campus de Beaulieu, 35042 Rennes, France \\ dauge@univ-rennes1.fr, URL: http://www.maths.univ-rennes1.fr/ dauge
}

\section{Maxwell eigenfrequency problem}

The problem consists in finding the non-zero frequencies $\omega>0$ such that there exists an electromagnetic field $(\mathbf{E}, \mathbf{H}) \neq 0$ solving the equation

$$
\operatorname{curl} \varepsilon \mathbf{E}-i \omega \mu \mathbf{H}=0 \quad \text { and } \quad \operatorname{curl} \mu \mathbf{H}+i \omega \varepsilon \mathbf{E}=0 \quad \text { in } \quad \Omega,
$$

in the cavity $\Omega$. We assume perfect conductor boundary conditions, that is $\mathbf{E} \times \mathbf{n}=0$ and $\mathbf{H} \cdot \mathbf{n}=0$ on $\partial \Omega$, where $\mathbf{n}$ denotes the outer unit normal on $\partial \Omega$. Concentrating on the problems posed by the singularities of the boundary of $\Omega$, we assume that the dielectric material filling $\Omega$ is homogeneous and isotropic, that is, after a possible change of unknowns $\varepsilon=\mu=1$.

We want to solve (1) by a Galerkin procedure. The first step is to propose a variational formulation for (1). Eliminating $\mathbf{H}$ from the equations, we find the formulation

$$
\begin{aligned}
& \mathbf{E} \in H_{0}(\operatorname{curl} ; \Omega), \quad \forall \mathbf{E}^{\prime} \in H_{0}(\operatorname{curl} ; \Omega), \\
& \qquad \int_{\Omega} \operatorname{curl} \mathbf{E} \cdot \operatorname{curl} \mathbf{E}^{\prime} \mathrm{d} x=\omega^{2} \int_{\Omega} \mathbf{E} \cdot \mathbf{E}^{\prime} \mathrm{d} x,
\end{aligned}
$$

where $H_{0}(\mathbf{c u r l} ; \Omega)$ is the space of $L^{2}(\Omega)$ fields $\mathbf{E}$ with curl $\mathbf{E} \in L^{2}(\Omega)$ and tangential component $\mathbf{E} \times \mathbf{n}=0$ on $\partial \Omega$. Testing against gradients of functions $\varphi \in H_{0}^{1}(\Omega)$ we find that if $\omega \neq 0$, then $\operatorname{div} \mathbf{E}=0$. Conversely, any such gradient is a solution of (2) with $\omega=0$.

There are two classes of strategies to overcome the difficulties generated by the presence of this big kernel:

(i) Discretize (2) directly by special families of finite elements possessing a discrete deRham complex property: these are the edge elements first introduced by NEDELEC $[3,4]$.

(ii) Regularize (2) by a term $\langle$ div, div $\rangle$ and use more standard elements.

Strategy (i) is widely spread but, still, it is worthwhile to develop an alternative, which will have different advantages. 
At first glance, strategy (ii) is a very bad idea if $\Omega$ has non-convex corners or edges. The reason for this is now well known: The variational space for the continuous problem is

$$
X_{N}=\left\{\mathbf{E} \in H_{0}(\operatorname{curl} ; \Omega), \quad \operatorname{div} \mathbf{E} \in L^{2}(\Omega)\right\}
$$

whereas any finite element field which is conforming for the bilinear form $\langle\text { curl, curl }\rangle_{L^{2}}+\langle\operatorname{div}, \operatorname{div}\rangle_{L^{2}}$ is continuous across the faces of the elements, therefore belong to the subspace $H_{N} \subset X_{N}$ of $H^{1}(\Omega)$ fields. But $H_{N}$ is closed in $X_{N}$ for the norm $\|\operatorname{curl}\|_{L^{2}}+\|\operatorname{div}\|_{L^{2}}$ and finally $H_{N} \neq X_{N}$ as soon as $\Omega$ has reentrant corners in $2 \mathrm{D}$ and reentrant edges in $3 \mathrm{D}$. Thus we are in the somewhat unusual situation where the convergence of the Galerkin method does not mean that the limit is the solution of the continuous problem.

\section{Weighted regularization}

In order to overcome this difficulty due to the lack of density, a weighted regularization was proposed in [1], that is replacing the regularization term $\langle\operatorname{div}, \operatorname{div}\rangle_{L^{2}}$ by the scalar product $\langle\operatorname{div}, \operatorname{div}\rangle_{Y}$ in another functional space $Y$ containing $L^{2}(\Omega)$ and contained in $H^{-1}(\Omega)$. Such a space can be conveniently realized as a weighted $L^{2}$ space, where the weight is a power $\gamma, 0 \leq \gamma \leq 1$, of a distance function $\underline{d}: x \mapsto \underline{d}(x)$ to the reentrant corners or edges of $\Omega$. The variational space is, instead of (3)

$$
X_{N}[\gamma]=\left\{\mathbf{E} \in H_{0}(\mathbf{c u r l} ; \Omega), \quad \underline{d}(x)^{\gamma} \operatorname{div} \mathbf{E} \in L^{2}(\Omega)\right\}
$$

and the corresponding variational formulation is (with $\alpha:=2 \gamma$ and $s>0$ chosen ad libitum)

$$
\begin{aligned}
\mathbf{E} \in X_{N}[\gamma], \quad \forall \mathbf{E}^{\prime} \in X_{N}[\gamma] \\
\quad \int_{\Omega}\left(\operatorname{curl} \mathbf{E} \cdot \operatorname{curl} \mathbf{E}^{\prime}+s \underline{d}(x)^{\alpha} \operatorname{div} \mathbf{E} \operatorname{div} \mathbf{E}^{\prime}\right) \mathrm{d} x=\omega^{2} \int_{\Omega} \mathbf{E} \cdot \mathbf{E}^{\prime} \mathrm{d} x
\end{aligned}
$$

It is clear that the eigenpairs $(\mathbf{E}, \omega)$ with $\omega \neq 0$ of $(2)$ are eigenpairs of (5). Any $\operatorname{grad} \varphi$ with $\varphi \in H_{0}^{1}(\Omega)$ is an eigenvector of (2) with $\omega=0$. For problem (5) also the "spurious" eigenvectors are gradients $\operatorname{grad} \varphi$ with $\varphi \in H_{0}^{1}(\Omega)$ and $\underline{d}^{\gamma} \Delta \varphi \in L^{2}(\Omega): \varphi$ is such that $\underline{d}^{\gamma} \varphi$ is an eigenvector of the self-adjoint operator $A[\gamma]:=\underline{d}^{\gamma} \Delta \underline{d}^{\gamma}$ with Dirichlet conditions. One can prove

Theorem 1. The eigenpairs (E, $\omega)$ of problem (5) can be split into two types (i) $\operatorname{div} \mathbf{E}=0$ and $\omega \neq 0:(\mathbf{E}, \omega)$ is then a Maxwell eigenpair.

(ii) $\operatorname{curl} \mathbf{E}=0$ and $\omega^{2}=s \nu$ with $\nu$ and eigenvalue of the operator $A[\gamma]$.

It is important to know that the spectrum of $A[\gamma]$ is separated from 0 : 
Theorem 2. The eigenvalues of $A[\gamma]$ decrease when $\gamma$ increases.

For any $\gamma \leq 1, A[\gamma]$ is positive definite.

For any $\gamma<1, A[\gamma]$ has a compact resolvent.

From Theorems 1 and 2 we know that the continuous formulation (5) can be used to determine the Maxwell eigenvalues for any $0 \leq \alpha \leq 2$. Now, we have to investigate whether there exists $\alpha$ so that smooth fields are dense in $X_{N}[\gamma]$. The following statement is deduced from [1]

Theorem 3. Let $\Omega$ be non-convex polygon or a non-convex polyhedron. Then there exists $\gamma_{\Omega}<1$ such that for any $\gamma, \gamma_{\Omega}<\gamma<1$, the space $H_{N}$ of $H^{1}$ vector fields satisfying the electric boundary condition is dense in $X_{N}[\gamma]$. If $\Omega$ is a polygon, let $\omega_{\Omega}$ be its largest corner. Then $\gamma_{\Omega}=1-\pi / \omega_{\Omega}$.

There holds a similar formula for polyhedra. Moreover, it is proved in [1] that for the source problem in a polygon, if nodal elements of degree $p \geq 5$ are used, we have a convergence rate in energy norm for the $h$-version of finite elements in $h^{\tau-\varepsilon}$ for all $\varepsilon>0$, where

$$
\tau=\min \left\{\gamma-1+\frac{\pi}{\omega_{\Omega}},-1+\frac{\pi}{\omega_{\text {conv }}}, \frac{\pi}{\omega_{\Omega}}\right\},
$$

with $\omega_{\text {conv }}$ the largest convex angle of $\Omega$.

\section{Experiments in an L-shape domain}

The condition $p \geq 5$ is not necessary for practical convergence of the method (it was simply comfortable for the proof). On the other hand, owing to the classical structure of the singularities (though unbounded) we can guess that a geometrical refinement of the mesh near singular points of the boundary will improve the performance of the method.

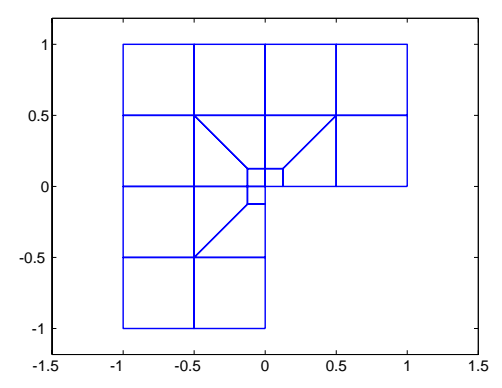

3 layers.

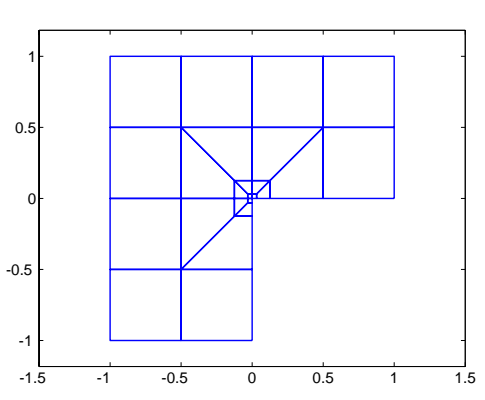

4 layers.

Fig. 1. Meshes on the L-shape domain

Figure 1 presents our first domain of computation $\Omega$ with the first two levels of refinement of the mesh. Here we use a ratio 4 . 
We have computed the first Maxwell eigenvalue by a Galerkin discretization of formulation (5) with $\alpha=2$ in eight nested meshes where the number of layers increases from 3 to 10 and with nodal elements based on tensor $\mathbb{Q}_{p}$ polynomials, $p$ ranging from 1 to 10 . Note that, since the edges of the boundary $\partial \Omega$ are straight, the essential boundary condition $\mathbf{E} \times \mathbf{n}=0$ is implemented exactly. The computations are done with the Finite Element Library MÉLINA [2].

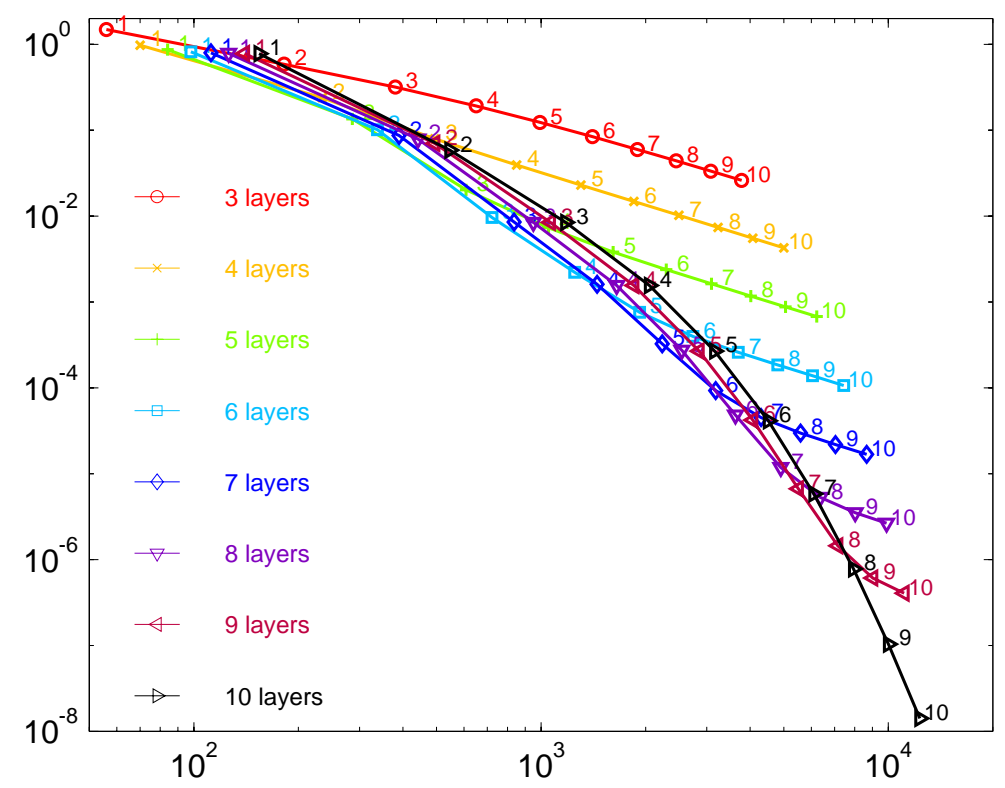

Fig. 2. Relative errors for $1^{\text {st }}$ eigenvalue

In Figure 2 relative errors for the first Maxwell eigenvalue are represented: The comparison value $\omega^{2}=1.4756218241$ is obtained by solving the Neumann problem on the same domain with the finest mesh and $p=10$ - the 2D Maxwell computations work as a benchmark problem since the Maxwell eigenpairs are the $(\operatorname{curl} \varphi, \sqrt{\nu})$ with $\varphi$ eigenvector of the Laplace Neumann problem with eigenvalue $\nu$.

The abscissae represent the number of degrees of freedom, and the ordinates the relative error. Each line connects the relative errors for a fixed number of layers and as $p$ goes from 1 to 10 . The numbers above the lines indicate the values of the degree $p$.

In Figure 3 we represent the convergence rates of the previous relative errors with respect to the number $N$ of degrees of freedom $(N$ is proportional to $p^{2}$ on each mesh) and as $p$ increases. The $h p$ effect is clearly visible. 


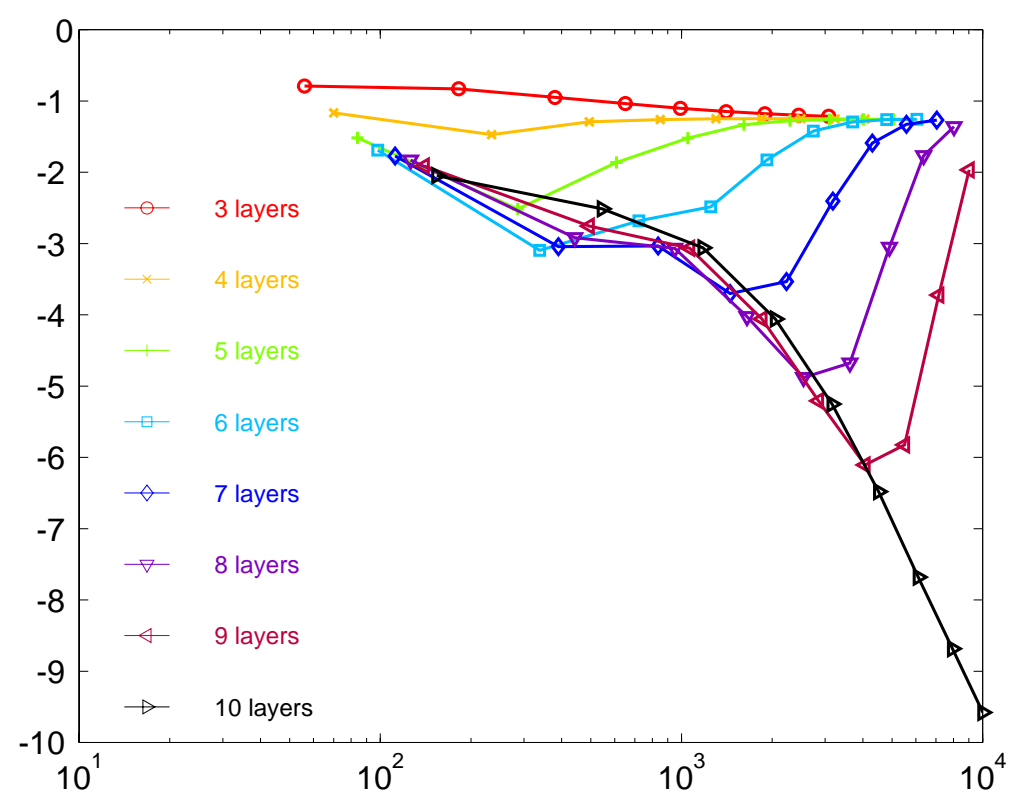

Fig. 3. Convergence rates of relative errors

\section{Experiments in a curved L-domain}

The domain $\Omega$ with curved boundaries (arcs of circle $A_{1}, A_{2}$ and $A_{3}$ of radii 1,2 and 3 respectively) for which we did the computations is represented opposite. The discretization of its boundary depends now on the mesh we choose and on the degree $q$ of the geometrical approximation.

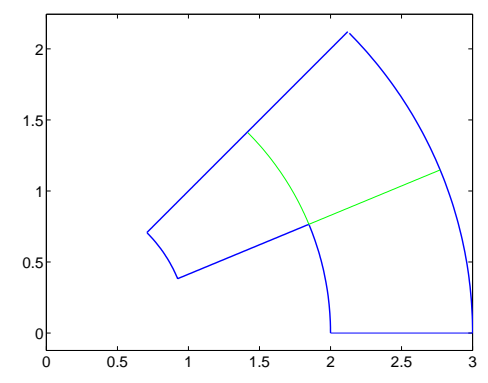

Fig. 4. The curved L-domain

The meshes are similar to those constructed in the straight L-domain of Section 3 and refined by adding successive layers of elements around the reentrant corner. Let $m$ be the number of layers of the mesh. Whatever the value of $m$ we have 4 elements abutting at the exterior $\operatorname{arc} A_{3}$, and 2 at the interior arc $A_{1}$. Along $A_{2}$ there are $m$ elements. We use a geometrical approximation of degree $q$ ranging from 1 to 6 , independently of the functional 
degree $^{1} p$. The values of $q$ and $m$ determine different approximations $\Omega_{q, m}$ of $\Omega$, which consist in defining each element by a grid of $(q+1)^{2}$ of GaussLobatto points. For example, when $q=1$, the discretized domains $\Omega_{1, m}$ are polygonal approximations of $\Omega$, cf Figure 5 .
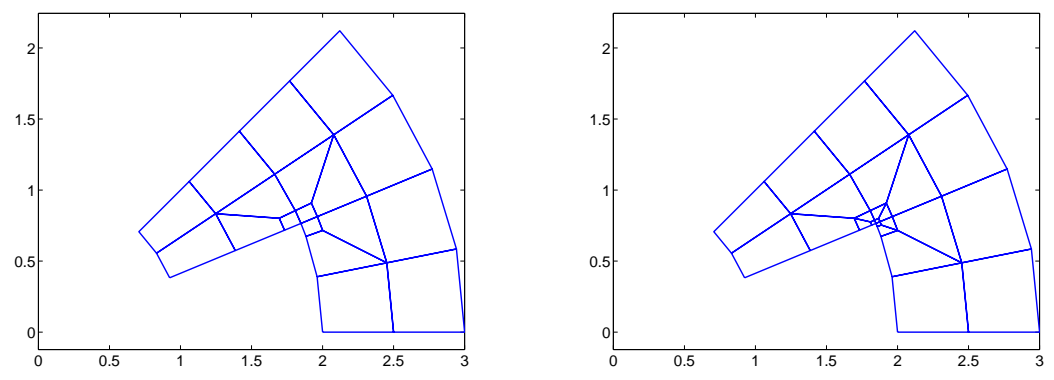

Fig. 5. Meshes on the approximate curved L-domains $\Omega_{q, m}, q=1, m=3,4$

If we represent the relative errors for the first eigenvalue with the eight best geometrical approximations $\Omega_{6, m}$ with $m=3, \ldots, 10$, as $p$ increases, we obtain a graph very similar to Figure 2 .

We prefer to investigate the influence of the geometrical degree $q$ on the quality of the approximation. We represent in Figures 6 and 7 the relative errors obtained by the computation of the first and second Maxwell eigenvalues with the variational formulation (5) on the meshes with 10 layers and geometrical degree $1 \leq q \leq 6$. The comparison is done with the Neumann eigenvalues computed on the same mesh with $q=6$.

We have to note that our approximation is now non conforming for two reasons:

1. The domains of computation $\Omega_{q, m}$ do not coincide exactly with $\Omega$ : the boundary is approximated by piecewise curves of degree $q$. Only at the grid points $a_{i}$ the boundary $\partial \Omega_{q, m}$ of the approximate domain coincides with $\partial \Omega$.

2. The essential boundary condition $\mathbf{E} \times \mathbf{n}=0$ is implemented only at the functional (Gauss-Lobatto) nodes $b_{j}$. If and only if $q=1$, these pointwise conditions imply that $\mathbf{E} \times \mathbf{n}=0$ on $\partial \Omega_{q, m}$.

We can see the relative influence of the position of the geometrical nodes $a_{i}$ and the functional nodes $b_{j}$ by modifying their position. If the $a_{i}$ are defined from an equidistant grid and $b_{j}$ unchanged, the results are very similar.

\footnotetext{
${ }^{1}$ The geometrical degree $q$ is the degree of the local maps which transform the reference element $\hat{K}$ into the elements $K$. The functional degree $p$ is the degree of the interpolating polynomials in $\hat{K}$ for the test and trial functions. The case $p=q$ corresponds to isoparametric elements.
} 


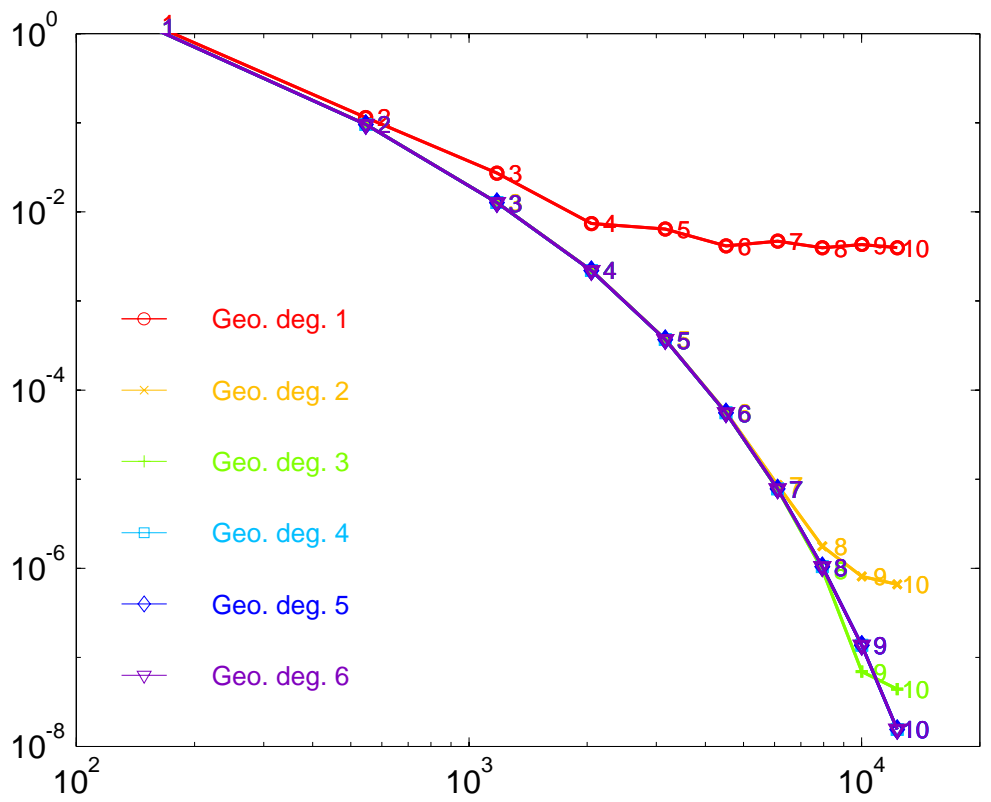

Fig. 6. Relative errors for the $1^{\text {st }}$ eigenvalue

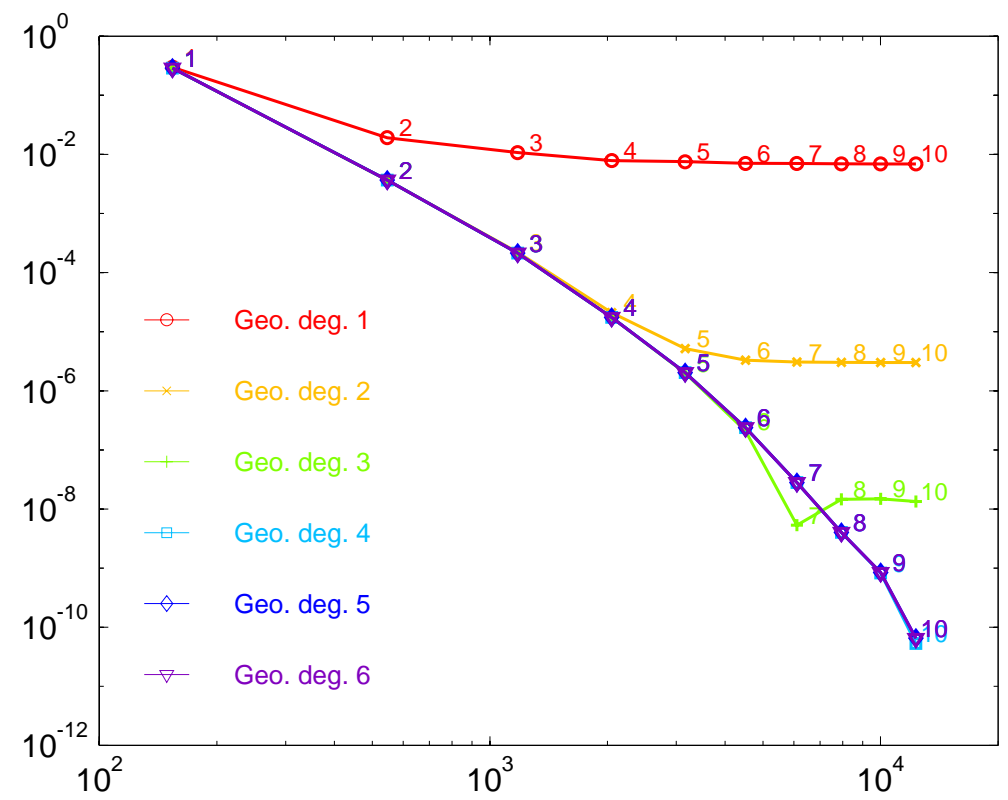

Fig. 7. Relative errors for the $2^{\text {nd }}$ eigenvalue 
If, on the other hand, the $a_{i}$ are Gauss-Lobatto points and the $b_{j}$ are equidistant, we obtain for the second eigenvalue the numerical results contained in Figure 8.

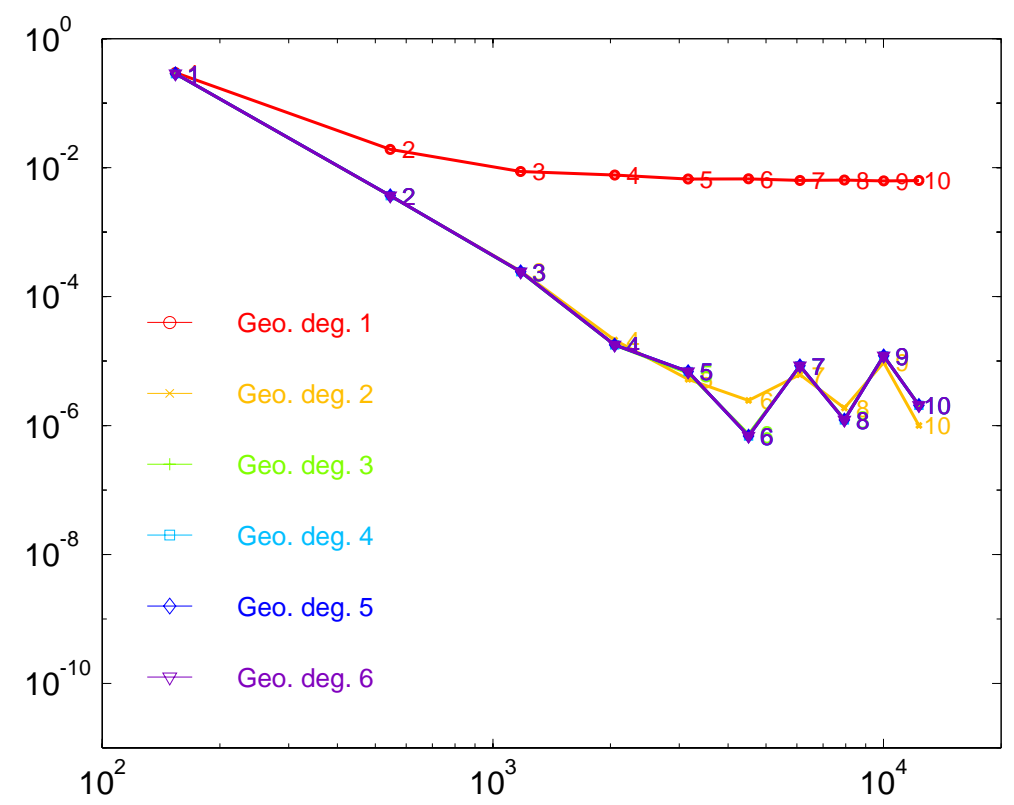

Fig. 8. Relative errors for the $2^{\text {nd }}$ eigenvalue with equidistant functional nodes

We see that the choice of equidistant nodes for the interpolation of the boundary condition $\mathbf{E} \times \mathbf{n}=0$ is a very serious "variational crime" that prevents further reduction of the error for degrees $p \geq 5$. Note that this phenomenon is only observed for vector boundary conditions and curved boundaries. It does not appear in scalar problems nor on domains with piecewise straight boundaries.

\section{References}

1. M. Costabel, M. Dauge. Weighted regularization of maxwell equations in polyhedral domains. Preprint 01-26, Université de Rennes 1 (2001). To appear in Numer. Math.

2. D. Martin. Mélina. On line documentation: http://www . maths . univ-rennes $1 . f r / \sim$ dmartin.

3. J.-C. NÉDÉLEC. Mixed finite elements in $\mathbb{R}^{3}$. Numer. Math. 35 (1980) 315-341.

4. J.-C. NÉDÉLEC. A new family of mixed finite elements in $\mathbb{R}^{3}$. Numer. Math. 50(1) (1986) 57-81. 